\title{
Sleeve gastrectomy: the ideal option for metabolic surgery?
}

Ricardo Cohen

I congratulate Michel Gagner for a wellarticulated argument favouring sleeve gastrectomy (SG) as 'the ideal' weight loss procedure in his News and Views article (Sleeve gastrectomy - the ideal choice for weight-loss surgery. Nat. Rev. Endocrinol. doi:10.1038/nrendo.2013.100). ${ }^{1}$ Although long-term data are still scarce, in the short to mid-term (1-3 years), weight loss after SG seems to be higher of that achieved after gastric banding and similar to Rouxen-Y gastric bypass (RYGB). ${ }^{2}$ The question is, however, if this is sufficient evidence to define SG as the 'ideal' choice. I am afraid that this is not the case.

Unlike bariatric surgery, conceived as a weight loss intervention, metabolic surgery is based on treating obesity and type 2 diabetes mellitus (T2DM) from the perspective of metabolic illnesses. As recently shown by Rubino et al., such change in end points has several ramifications, distinguishing metabolic from bariatric surgery. Consistent with the intent to treat a disease, not weight per se, the choice of the intervention should be based on mechanisms of action and metabolic outcomes, not simply on weight loss.

Although both SG and RYGB share similar effects on certain mechanisms of glucose homeostasis (incretin levels), there is experimental evidence that re-routing the food through the gastrointestinal tract, excluding the proximal bowel (as is the case with RYGB), promotes specific, weight-independent effects on T2DM. ${ }^{3-5}$ In a recent randomized study comparing RYGB versus SG versus medical therapy in diabetic patients, Kashyap et al. ${ }^{6}$ found that 2 years after surgery, RYGB induced greater improvement of insulin sensitivity (Matsuda Index) and $\beta$-cell function (insulin disposition index), compared with SG, whereas weight loss was similar for both procedures. While a few clinical observations have suggested that the effect of SG on glucose homeostasis is similar to that of RYGB, ${ }^{7,8}$ all available randomized clinical trials (RCT) with T2DM control as a primary end point showed better outcomes regarding T2DM remission after RYGB compared with SG. ${ }^{9,10}$ In a RCT with diabetic patients, comparing SG and minigastric bypass (which involves duodenal exclusion), the latter procedure resulted in twice the number of T2DM remissions, with similar weight loss after both operations. ${ }^{9,11}$ Another RCT also showed that while both SG and RYGB substantially improved T2DM $\left(\mathrm{HbA}_{1 \mathrm{c}}<6 . \%\right.$, with or without meds) in $37 \%$ and $42 \%$ respectively, no patients after RYGB required pharmacologic treatment to maintain normoglycaemia whereas most of SG patients needed drugs. ${ }^{12}$ The current knowledge on mechanisms of action of gastrointestinal procedures and the evidence from RCTs suggest that bypass operations may be more effective than SG in improving glycemic control. We are in the dawn of a new discipline, metabolic surgery, where reporting outcomes based on weight loss only is not the primary end point anymore. Of course, it is appreciated, but we can safely state that weight loss should not be considered as the sole means to control T2DM, but rather serve as an added benefit of interventions aimed at improving T2DM (including diet and surgery). SG may be a good weight loss procedure, but it has yet to prove its effects over glycemic control beyond weight loss, and should not be offered as a primary procedure focusing T2DM remission.

The Center of Excellence for Metabolic and Bariatric Surgery, Hospital Oswaldo Cruz, Rua Padre João Manuel, 222 \#130, 01411-000 São Paulo, Brazil. ricardo.cohen@haoc.com.br
Competing interests

The author declares no competing interests.

1. Gagner, M. Obesity: Sleeve gastrectomy - the ideal choice for weight-loss surgery. Nat. Rev. Endocrinol. 9, 382-384 (2013).

2. Brethauer, S. A. Sleeve gastrectomy. Surg. Clin. North Am. 91, 1265-1279 (2011).

3. Patel, R. T., Shukla, A. P., Ahn, S. M., Moreira, M. \& Rubino, F. Surgical control of obesity and diabetes: The role of intestinal vs gastric mechanisms in the regulation of body weight and glucose homeostasis. Obesity http://dx.doi.org/10.1002/oby.20441.

4. Breen, D. M. et al. Jejunal nutrient sensing is required for duodenal-jejunal bypass surgery to rapidly lower glucose concentrations in uncontrolled diabetes. Nat. Med. 18, 950-955 (2012).

5. Salinari, S. et al. Jejunal proteins secreted by $\mathrm{db} / \mathrm{db}$ mice or insulin-resistant humans impair the insulin signaling and determine insulin resistance. PLOS ONE 8, e56258 (2013).

6. Kashyap, S. R. et al. Metabolic effects of bariatric surgery in patients with moderate obesity and type 2 diabetes: analysis of a randomized control trial comparing surgery with intensive medical treatment. Diabetes Care $\mathbf{3 6}$ 2175-2182 (2013).

7. Peterli, R. et al. Improvement in glucose metabolism after bariatric surgery: comparison of laparoscopic Roux-en-Y gastric bypass and laparoscopic sleeve gastrectomy: a prospective randomized trial. Ann. Surg. 250, 234-241 (2009).

8. Leonetti, F. et al. Obesity, type 2 diabetes mellitus, and other comorbidities: a prospective cohort study of laparoscopic sleeve gastrectomy vs medical treatment. Arch. Surg. 147, 694-700 (2012).

9. Lee, W.-J. et al. Gastric bypass vs sleeve gastrectomy for type 2 diabetes mellitus: a randomized controlled trial. Arch. Surg. 146, 143-148 (2011).

10. Garrido-Sanchez, L. et al. Bypass of the duodenum improves insulin resistance much more rapidly than sleeve gastrectomy. Surg. Obes. Relat. Dis. 8, 145-150 (2012).

11. Lee, W.-J. et al. Laparoscopic sleeve gastrectomy for diabetes treatment in nonmorbidly obese patients: Efficacy and change of insulin secretion. Surgery 147, 664-669 (2010).

12. Schauer, P. R. et al. Bariatric surgery versus intensive medical therapy in obese patients with diabetes. N. Engl. J. Med. 366, 1567-1576 (2012) 